\title{
Prospective clinical evaluation of chairside-fabricated zirconia-reinforced lithium silicate ceramic partial crowns-5-year results
}

\author{
Sven Rinke ${ }^{1} \mathbb{D} \cdot$ Tanja Zuck $^{1} \cdot$ Tim Hausdörfer $^{2} \cdot$ Andreas Leha $^{3} \cdot$ Torsten Wassmann $^{1} \cdot$ Dirk Ziebolz $^{4}$
}

Received: 17 December 2020 / Accepted: 6 August 2021 / Published online: 20 August 2021

(c) The Author(s) 2021

\begin{abstract}
Objectives A university-based randomized clinical study evaluated the 5-year performance of chairside-fabricated zirconiareinforced lithium silicate (ZLS)-ceramic partial crowns.

Material and methods Forty-five patients were restored with 61 chairside-fabricated ZLS-restorations (Cerec SW 4.2, Dentsply Sirona, Germany; Vita Suprinity, Vita Zahnfabrik, Germany). Deviating from the manufacturers' recommendations, restorations with reduced minimum material thicknesses (MMT) were fabricated: group 1, MMT $=0.5-0.74 \mathrm{~mm}(n=31)$; group 2, MMT $=0.75-1.0 \mathrm{~mm}(n=30)$. For luting, a self-adhesive cement (SAC) or a total-etch technique with a composite cement (TEC) was applied. Statistical evaluation was performed by time-to-event analysis (Kaplan-Meier). Possible covariates of the survival (SVR) and success rates (SCR), evaluated in a Cox regression model, were MMT, restoration position (premolar/molar), and cementation technique (SAC vs. TEC).

Results Forty patients (54 restorations, premolars, $n=23$; molars, $n=31$ ) participated in the 5-year follow-up. Five losses due to ceramic fractures occurred in group $1(n=28)$ (SVR: 83.0\% [95\% confidence interval (CI): 0.71-0.96]). Group 2 $(n=26)$ showed no losses (SVR: 100\%). The success rate for partial crowns placed on premolars was 100\% and 69\% (95\% CI: 0.54-0.84) for molar restorations. Recementation was required in 4 restorations with SAC (SCR: 86\% [95\% CI: 0.73-0.99]; SCR-DC: $100 \%)$. Restorations in group 2 showed a significantly reduced risk of material fracture hazard ratio $(\mathrm{HR})=0.09$, $p=0.0292)$ compared with the restorations in group 1 . Molar partial crowns showed an increased risk for a clinical intervention $(\mathrm{HR}=5.26, p=0.0222)$ compared to premolar restorations.

Conclusions Material thickness and position of the restoration are risk factors influencing the survival and success rate of ZLS-ceramic partial crowns.

Clinical relevance Observation of an MMT of at least $0.75-1.0 \mathrm{~mm}$ for ZLS-ceramics is essential to avoid material-related fractures.
\end{abstract}

Clinical trial registration: German Clinical Trails Register (trial number: DRKS00005611)

Keywords High-strength glass-ceramics $\cdot$ Partial crown $\cdot$ Survival rate $\cdot$ Success rate $\cdot$ Clinical study

Sven Rinke

sven.rinke@med.uni-goettingen.de

1 Department of Prosthodontics, University Medical Center, Robert-Koch-Str. 40, 37075 Goettingen, Germany

2 Department of Preventive Dentistry, Periodontology, and Cariology, University Medical Center, Goettingen, Germany

3 Department of Medical Statistics, University Medical Center, Goettingen, Germany

4 Department of Cariology, Endodontology and Periodontology, University Medical Center, Leipzig, Germany

\section{Introduction}

In patients with extensively destroyed natural teeth, a partial crown restoration can be a minimally invasive alternative to full crowns [1,2]. Apart from resin-based materials, dental ceramics are the group of materials with the longest clinical history of use for the fabrication of tooth-colored partial crowns [3, 4]. The available longterm clinical documentation indicates that up to now, a material fracture is the most frequent cause of the loss of all-ceramic partial crowns, even those fabricated according to the manufacturer's recommendation with a minimum 
material thickness (MMT) of $1.5 \mathrm{~mm}$ [5-8]. This observation is based on the fact that in these studies, mostly feldspathic or leucite-reinforced glass-ceramics with a mean flexural and tensile strength of less than $200 \mathrm{MPa}$ were used $[5,7]$. Thus, dental ceramic materials with improved mechanical properties offer the possibility of reducing the incidence of material-related failure [9].

High-strength glass-ceramics, such as lithium disilicate ceramics or newly developed zirconia-reinforced lithium silicate (ZLS) ceramics with a flexural and tensile strength of 370-450 MPa, provide stability 2- to 3-times greater than that of leucite-reinforced glass-ceramics [10-13]. Clinical studies with mean observational periods of 11 years have demonstrated that the improved mechanical properties of lithium disilicate materials led to significantly reduced material-related failure rates of ceramic onlays and partial crowns [14-16]. Nevertheless, for the more recently introduced ZLS-ceramics, the evidence for a reduced fracture rate is still limited as of now because only studies with an observational period of up to 3 years are available [17-19]. Moreover, several in vitro studies have evaluated the effect of the MMT on the fracture characteristics of indirect all-ceramic restorations. These evaluations focused on determining whether the improved mechanical properties of the new high-strength ceramic materials allowed a reduction in the MMT [20-23]. Based on these results, several manufacturers have reduced the recommended material thickness for high-strength glass-ceramic restorations to $1 \mathrm{~mm}$.

However, it must be considered that the reasons for the failure of all-ceramic restorations are multifactorial. Apart from the material, fabrication technique, and type of cementation, patient-related factors, such as bruxism or location of the restoration, have had clinically relevant effects on the long-term durability of all-ceramic restorations [24-26].

A general recommendation for a reduced MMT for allceramic restorations made of high-strength glass-ceramics should therefore be based on clinical studies. These studies are still sparse. Therefore, clinical data from additional studies are mandatory to generate an evidence-based recommendation $[18,27]$.

The present prospective clinical study aimed to evaluate the risk factors for the material-induced failure of chairside-produced (Cerec system, Dentsply Sirona, Bensheim, Germany) ZLS-ceramic partial crowns (Vita Suprinity, Vita Zahnfabrik, Bad Säckingen, Germany) with a special consideration of the applied cementation technique and a reduced MMT of 0.5 to $1.0 \mathrm{~mm}$. The following null hypothesis was formulated: The survival and success rates are independent of the cementation technique applied and the MMT of the restorations.

\section{Materials and methods}

\section{Patient recruitment}

Forty-five adult patients ( 28 female/17 male) with indications for a chairside-fabricated partial crown (covering all cusps) were included according to the following inclusion criteria:

- vital and symptom-free posterior teeth (premolar/ molar)

- existing antagonistic teeth

- at least one proximal contact.

Patients fulfilling the following criteria were excluded:

- Clinical symptoms of bruxism

- Preparation unsuitable for optical impression taking (e.g., deeply subgingival)

- Nonvital or endodontically treated teeth

- Untreated periodontal disease

- Age less than 18 years

A maximum of two partial crowns per patient was allowed. The study protocol was evaluated and approved by the ethics committee of the medical faculty of GeorgAugust University, Goettingen, Germany (No. 27/7/13). All patients participating in the study provided written informed consent.

\section{Treatment}

The clinical treatments and the manufacturing of the ceramic restorations were performed during a 6-month period (January-July 2014) by two experienced dentists in the Department of Preventive Dentistry, Periodontology, and Cariology, University Medical Center, Goettingen, Germany. All patients received professional prophylaxis including instructions for oral homecare procedures before the treatment. The preparation of the teeth followed the published recommendations for all-ceramic partial-crown restorations $[1,4,28]$.

After preparation, a powder-free intraoral scanning device (Cerec AC Omnicam, Dentsply Sirona, Bensheim, Germany) was used for optical impression taking. The monolithic partial crown restorations (Vita Suprinity, Vita Zahnfabrik, Bad Säckingen, Germany) were constructed with CAD software (Cerec software 4.2, Dentsply Sirona, Bensheim, Germany). They were fabricated in a wetmilling unit (Cerec MCXL, Dentsply Sirona, Bensheim, Germany). Subsequently, the restorations were crystallized 
and stained according to the manufacturer's instructions. After the try-in of the restoration, the intaglio surfaces of the restorations were conditioned with hydrofluoric acid (5\%, Vita Ceramics Etch, Vita Zahnfabrik, Bad Säckingen, Germany) for $20 \mathrm{~s}$ and then rinsed with water and dried with water-free and oil free air. Finally, all restorations were silanized (Monobond S, Ivoclar Vivadent, Schaan, Liechtenstein, exposure time: $1 \mathrm{~min}$ ).

The adhesive luting of the partial crowns was performed at random, either using a dual-curing composite cement (TEC; $n=30$ ) and multibottle bonding with the total-etch technique (Syntac classic and Variolink II, Ivoclar Vivadent, Schaan, Liechtenstein) or with a self-adhesive cement (SAC, RelyX Unicem, 3 M, Deutschland GmbH, Neuss, Germany; $n=31$ ), each with rubber dam application. In the SAC group, the prepared tooth was cleaned with a slurry of pumice, rinsed with water spray, dried shortly avoiding overdrying, and the luting agent was applied directly into the cavity without any further pretreatment of the hard tooth tissues. In the TEC group, the prepared tooth surfaces were treated with the total-etch technique using 37\% phosphoric acid gel condition the hard tissues (dentin 15 s/enamel $30 \mathrm{~s}$ ). The bonding agent (Syntac classic) was applied after the acid was rinsed off, and the preparations were carefully air-dried. The luting agent (Variolink II) was applied directly to the cavity. For the random assignment of participants to the cementation groups, an online statistical computing web program (www. randomization.com) was used to generate the randomization schedule.

After removing the excess cement, the luting agent of each restoration was polymerized for $120 \mathrm{~s}$. Then, occlusion was restored according to the initial situation (anteriorcanine guided occlusion or unilateral dynamic guidance).

However, because the standard setting for the MMT of the construction software (Cerec software 4.2) at the time of the clinical treatment phase was $0.7 \mathrm{~mm}$, all restorations were accidentally produced with a reduced MTT, thus violating the manufacturer's recommendations for the MMT being $1.0 \mathrm{~mm}$. This deviation from the original treatment protocol was only realized after the clinical treatment phase had been completed. The ethics committee was informed about this deviation from the study protocol and approved the continuation of the study with a modified study protocol, including the reduced MMT as a risk factor for the survival and success of the restorations. For this purpose, the generated construction data were analyzed regarding the MMT. Bucco-lingual cross sections of the construction data were analyzed with the measuring function of the Cerec SW. The minimum material thickness for all restorations occurred in the central fissure; therefore, this area was used to divide the fabricated ceramic partial crowns into two groups (Fig. 1).

Group 1: MMT in the central fissure of $0.5-0.74 \mathrm{~mm}$

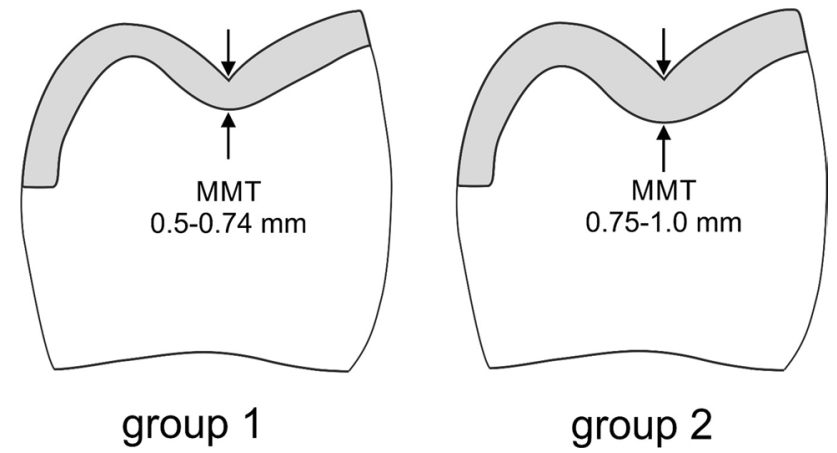

Fig. 1 Schematic of the preparation design and group distribution related to the occlusal minimum material thickness (MMT). Group 1: $\mathrm{MMT}=0.5-0.74 \mathrm{~mm}$. Group 2: MMT $=0.75-1.0 \mathrm{~mm}$

Fig. 2 a, b Clinical situation of an adhesively luted (TEC group) second lower premolar at the 5-year clinical evaluation: a. Occlusal view, b. buccal view. The restoration was rated "alpha" for the USPHS criteria marginal adaptation and marginal discoloration

a)

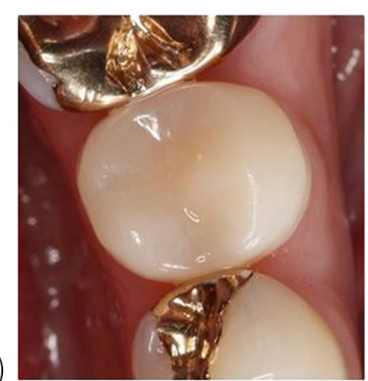

b)

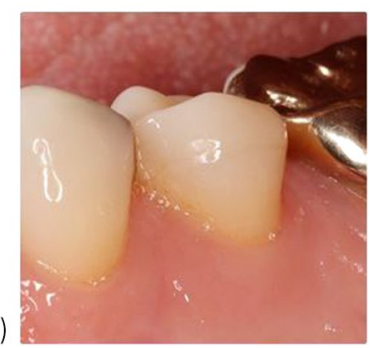

Group 2: MMT in the central fissure of $0.75-1.0 \mathrm{~mm}$

\section{Clinical evaluation}

The restorations were evaluated at the time of cementation (baseline) and followed by clinical examinations every 12 months using modified United States Public Health Service (USPHS) criteria that have been used in other clinical studies evaluating the clinical performance of ceramic partial crowns [29-31].

During the annual follow-up examinations, all restorations were clinically evaluated with a mirror, a number 9 dental probe (22,243.20; Stoma Dentalsysteme GmbH, Emmingen-Liptingen, Germany), and intraoral digital photographs. The vitality of the teeth was confirmed by $\mathrm{CO}_{2}$ testing. Every restoration was examined regarding fissures, fractures, loosening, caries; the following modified USPHS 
criteria were used to rate the marginal adaptation and marginal discoloration [30, 31] (Fig. 2a,b):

\begin{tabular}{|c|c|c|}
\hline \multirow[t]{4}{*}{ Marginal adaptation } & alpha & $\begin{array}{l}\text { Margin not discernible, probe } \\
\text { does not catch }\end{array}$ \\
\hline & bravo & $\begin{array}{l}\text { Probe catches on margin } \\
\text { but no gap; dentin or liner } \\
\text { exposed }\end{array}$ \\
\hline & charlie & $\begin{array}{l}\text { Probe catches on margin and } \\
\text { gap on probing, dentin or } \\
\text { liner exposed }\end{array}$ \\
\hline & delta & $\begin{array}{l}\text { Restoration fractured or } \\
\text { missing }\end{array}$ \\
\hline \multirow[t]{3}{*}{ Marginal discoloration } & alpha & No marginal discoloration \\
\hline & bravo & $\begin{array}{l}\text { Marginal discoloration, not } \\
\text { penetrated toward pulp }\end{array}$ \\
\hline & charlie & $\begin{array}{l}\text { Marginal discoloration pen- } \\
\text { etrated toward pulp }\end{array}$ \\
\hline
\end{tabular}

Failures that occurred before the examinations due to negative events (restoration loss, recementation if necessary, ceramic fracture, biological complications) were documented in the patient files and considered in the final results $[18,19]$.

The last follow-up examinations were performed between January and May 2019 by a trained dentist (T.P.) who was not involved in the placement. The training regarding the survival and success criteria [32] was performed by one of the authors (D.Z.) and repeated until each examiner had a substantial correlation, as measured by Cohen's Kappa $(k \geq 0.6)$.

\section{Statistical analysis}

The statistical analysis was based on information on the survival and success rates of the reconstructions. Survival was defined as the restoration being in situ at the time of the follow-up examination without signs of a total loss (i.e., the in situ criterion) [32]. A total loss was defined as a clinically unacceptable ceramic fracture of the restoration or a biological event (caries, tooth fracture, or periodontal disease) requiring complete replacement of the restoration or removal of the affected tooth. Success was defined as the reconstruction remaining unchanged and functional in situ without any intervention throughout the total observational period [32]. The survival time of a restoration was defined as the period between baseline (day of cementation) and either the last follow-up examination or, in the case of failure, the day of failure documentation in the patient file. The time-dependent survival rates of the restorations (based on the in situ criterion) and the success rates (intervention-free) of the partial crowns were calculated by Kaplan-Meier survival analysis.

The MMT $(0.5-0.74 \mathrm{~mm}$ vs. $0.75-1.0 \mathrm{~mm})$, the position of the restoration (premolar vs. molar), and the cementation technique (SAC vs. TEC) were evaluated as possible covariates of the time-dependent survival and success rates.

Different observations in the same patient (several partial crowns per patient) were evaluated as dependent based on the adapted estimation of variance in the Cox regression model. Therefore, a marginal model was applied for data analysis [33]. Univariate Cox regression was performed for every influencing factor. Penalized Firth correction was employed in the model in case of complete data separation. A $p$ value of less than 0.05 was accepted as statistically significant. Statistical analyses were performed with the statistical software R (version 3.5.3) using the R package "survival" (version 2.44.1.1) and the "prodim" module for the time-to-event analyses. The changes in the clinical criteria over time between baseline and the 5-year examination were evaluated separately for each luting procedure using the chisquare test $(\alpha=0.05)$.

A post hoc sample size calculation based on the incidence of prosthesis failures was conducted to determine the number of participants needed per group to confirm a statistically significant difference between the treatments with $\alpha=0.05$ and a power of $80 \%$.

\section{Results}

\section{Study population}

From January to June 2014, 45 patients were included in the present study and restored with a total of 61 partial crowns. One female patient withdrew her written consent for participation in the study at the time of the baseline examination, and these data were excluded. Four patients ( 1 female/3 male) with 5 molar restorations were lost during the followup period or declined further participation in the study. Two patients relocated from the area, one patient was unable to attend the last clinical follow-up due to severe illness, and one patient died. Their data were censored at the date of the last clinical evaluation or notification of a failure/intervention. Forty patients (26 female/14 male, recall rate: $90.9 \%$ ) with a total of 54 partial crowns participated in the 5-year follow-up examination. This clinical examination was carried out between January and May 2019 (mean observational period: $56 \pm 10$ months). Twenty-eight restorations were allocated to group 1 , and the remaining 26 restorations were assigned to group 2 . From the finally examined restorations, thirty-one were placed in the maxilla, and 23 were in the mandible ( 23 premolar and 31 molar partial crowns). Twenty-five of the examined restorations were luted adhesively by using the total-etch technique (TEC), and 29 partial crowns were luted with an SAC material. 


\section{Survival rate}

Five restorations failed completely during the 5-year followup period. The overall survival rate after 5 years was $91 \%$ (95\% confidence interval (CI): 0.84-0.98) (Fig. 3). All complete failures occurred in group $1(\mathrm{MMT}=0.5-0.74 \mathrm{~mm})$, and they were related to catastrophic material fractures involving the area where the MMT was measured (i.e., central fissure). Furthermore, all complete failures occurred for restorations placed on molars. The time-dependent 5-year survival rate was $83 \%$ (95\% CI: $0.71-0.96)$ in group 1 and $100 \%$ in group $2(\mathrm{MMT}=0.75-1.0 \mathrm{~mm})$.

With a hazard ratio (HR) of 0.09 , the Cox regression analysis showed an 11.1-fold reduced risk of material fracture in restorations with an MMT of $0.75-1.0 \mathrm{~mm}$ compared to restorations with an MMT of $0.5-0.74 \mathrm{~mm}$. This effect proved to be statistically significant $(p=0.029)$ (Figs. 4 and $5)$. The 5-year survival rate for ZLS partial crowns placed on molars was 85\% (95\% CI: 0.74-0.96) and 100\% for premolar restorations. The univariate Cox regression model revealed a 7.85-fold increased risk for a complete failure for molar restorations compared to premolar restoration. However, this effect proved not to be significant (0.0673). The survival rate of the partial crowns inserted with TEC and the totaletch technique was $93 \%$ (95\% CI: $0.85-1$ ), while the partial crowns inserted with SAC showed a survival rate of $90 \%$ (95\% CI: 0.8-1). The cementation technique (SAC vs. TEC, $\mathrm{HR}=1.43, p=0.677$ ) showed no significant influence on the survival rate of the partial crowns.

Therefore, the null hypothesis has to be partly rejected regarding the survival rate, as a significant effect of the

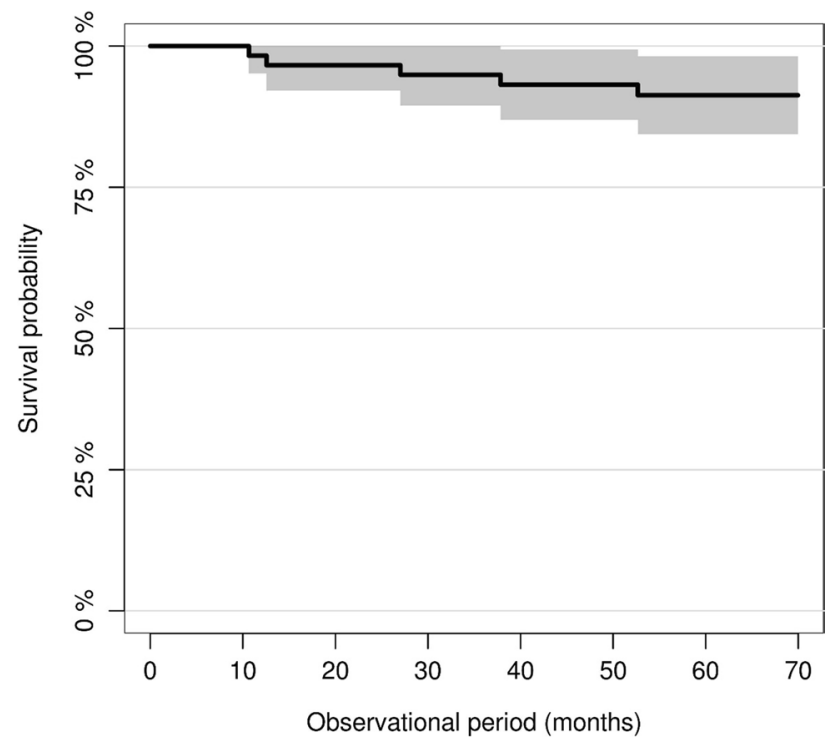

Fig. 3 Overall survival rate of the chairside-fabricated zirconia-reinforced lithium silicate (ZLS) partial crowns after a mean observational period of 5 years

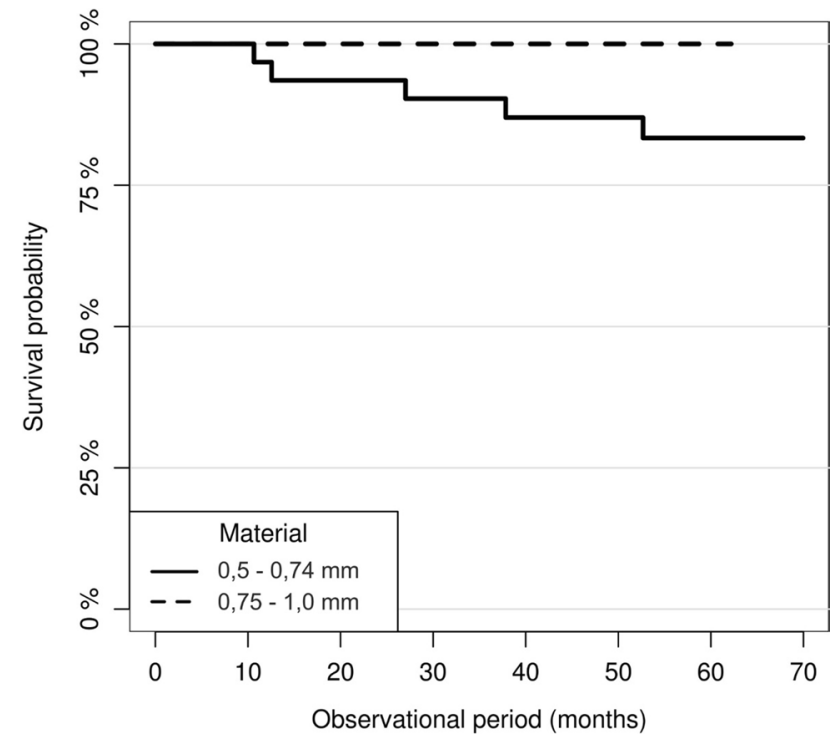

Fig. 4 Survival rate of the partial crown restorations by the occlusal minimum material thickness (MMT)

MMT could be demonstrated, while no effect of the cementation mode or the position of the restoration was detected.

\section{Success rate}

Apart from 5 total losses, six clinical interventions for six different restorations (6 patients) were necessary to maintain function. The time-dependent overall 5-year success rate (intervention-free survival) was $80.0 \%$ (95\% CI: 0.7-0.9) (Fig. 6). Four clinical interventions necessary to keep the restorations functional were caused by the loss of the retention of partial crowns inserted with SAC. The 4 restorations did not show any defects and were recemented with the same material. One additional intervention was caused by minor ceramic fractures $<2 \mathrm{~mm}^{2}$ (polishing) and the loss of pulp vitality (endodontic treatment). Further interventions due to temperature sensitivity, loss of vitality, or secondary caries were not necessary (100\% alpha ratings for the USHPS criteria "postoperative sensitivity", and "recurrent caries"). The specific success rate of restorations in group $2(\mathrm{MMT}=0.75-1.0 \mathrm{~mm})$ luted with the TEC material was $100 \%$.

Based on the Cox regression model, the overall success rate (intervention-free survival) was independent of

Fig. 5 Fracture of an ZLS partial crown from group A (MMT 0.5-0.74 mm). The fragments of the restoration were mobile at the date of the clinical examination

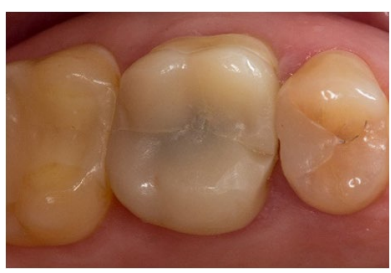




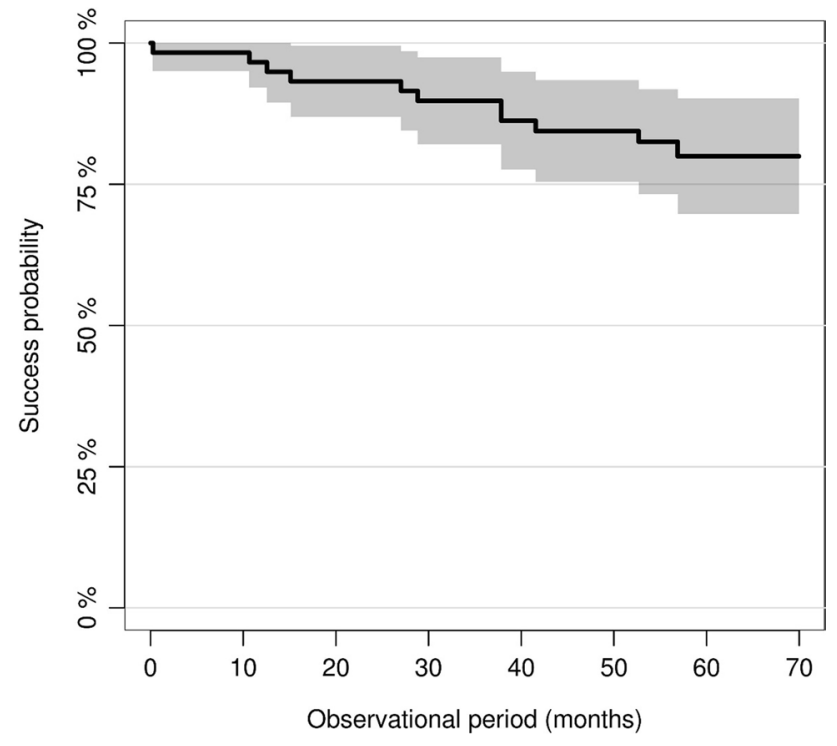

Fig. 6 Overall success rate of the chairside-fabricated zirconia-reinforced lithium silicate (ZLS) partial crowns over a mean observational period of 5 years

the MMT (0.5-0.74 $\mathrm{mm}$ vs. $0.75-1 \mathrm{~mm}, p=0.844)$, and the cementation technique $(p=0.124)$. The success rate for partial crowns placed on premolars was $100 \%$ and $69 \%$ (95\% CI: 0.54-0.84) for molar restorations. The univariate Cox regression revealed a 5.26-fold increased risk for partial crowns placed on molars for a failure or a clinical intervention compared to premolar restoration. This effect proved to be statistically significant ( $p=0.0222)$ (Fig. 7). The null hypothesis was partly rejected regarding the success rate.

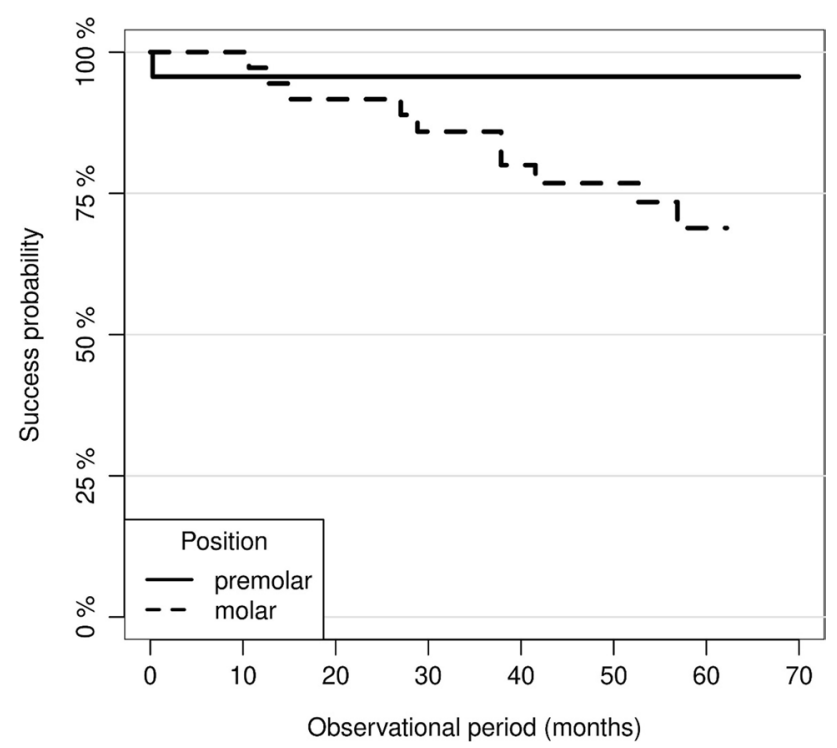

Fig. 7 Success probability of the partial crown restorations according to the tooth position
The specific 5-year success rate, referring to the event "loss of retention," was $86 \%$ (95\% CI: 0.73-0.99) for partial crowns inserted with SAC, whereas the success rate (no loss of retention) for the TEC-fixed restorations was 100\% (Fig. 8).

Related to the event loss of retention, partial crowns that were cemented with SAC showed a 9.2-fold greater risk of requiring recementation than restorations cemented with TEC. Although this association was not statistically significant $(p=0.0527)$, it shows a strong tendency toward an influence of the cementation technique on the success rate of partial crowns.

At baseline, the marginal adaptation was rated alpha in 29 restorations (96.7\%) in group TEC and 27 restorations (93.1\%) in group SAC. All other restorations were rated bravo. At the 5-year recall, the alpha ratings decreased to $36.0 \%$ in group TEC and to $23.1 \%$ in group SAC (Table 1 ).

At baseline, the marginal discoloration was rated alpha for all restorations in both groups. Until the 5-year clinical examination, the alpha ratings decreased to $56.0 \%$ (group TEC), resp. $46.2 \%$ (group SAC). Regarding marginal adaptation and marginal discoloration, a statistically significant difference $(p<0.05)$ could be found in both groups for the data assessed at baseline and after 5 years (Table 1).

Regarding the parameters "marginal adaptation" and "marginal discoloration," no significant differences for the data assessed at the 5-year recall were detected between the restorations in group TEC and group SAC.

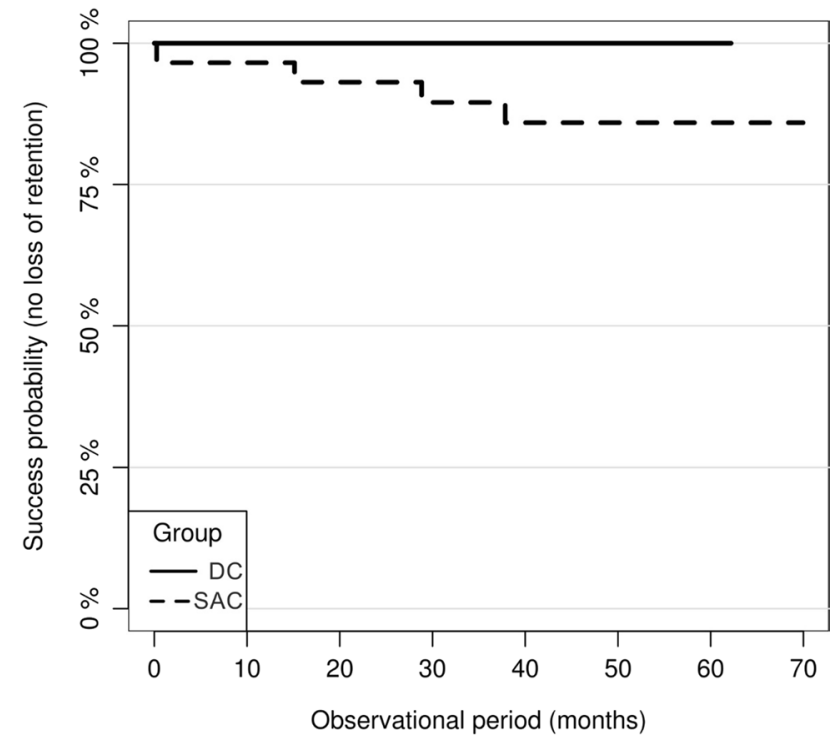

Fig. 8 Success probability of the partial crown restorations based on the loss of retention according to the cementation technique. TEC: total-etch technique with dual-curing cement, SAC: self-adhesive cement 
Table 1 Ratings for selected USPHS criteria at baseline, 2-year, and 5-year clinical examination

\begin{tabular}{lllllllll}
\hline & \multicolumn{3}{c}{ Marginal adaptation $^{4}$} & \multicolumn{4}{c}{ Marginal discoloration $^{4}$} \\
\hline Group & Time & alpha & bravo & charlie & delta & alpha & bravo & charlie \\
TEC & Baseline $(n=30)$ & $29(96.7 \%)$ & $1(3.3 \%)$ & 0 & 0 & $30(100 \%)$ & 0 & 0 \\
& 2 years $(n=28)^{1}$ & $23(82.1 \%)$ & $5(17.9 \%)$ & 0 & 0 & $21(75.0 \%)$ & $7(25.0 \%)$ & 0 \\
& 5 years $(n=25)^{2}$ & $9(36.0 \%)$ & $15(60.0 \%)$ & $1(4.0 \%)$ & 0 & $14(56.0 \%)$ & $6(24.0 \%)$ & $5(20.0 \%)$ \\
SAC & Baseline $(n=29)$ & $27(93.1 \%)$ & $2(6.9 \%)$ & 0 & 0 & $29(100 \%)$ & 0 & 0 \\
& 2 years $(n=29)$ & $21(72.4 \%)$ & $8(27.6 \%)$ & 0 & 0 & $20(69.0 \%)$ & $9(31.0 \%)$ & 0 \\
& 5 years $(n=26)^{3}$ & $6(23.1 \%)$ & $18(69.2 \%)$ & $2(7.7 \%)$ & 0 & $12(46.2 \%)$ & $7(26.9 \%)$ & $7(26.9 \%)$ \\
\hline
\end{tabular}

${ }^{1}$ Only 28 restorations remained because two partial ceramic crowns failed prior to the follow-up examination

${ }^{2}$ Only 25 restorations remained because three patients did not attend follow-up examinations

${ }^{3}$ Only 26 restorations remained because three partial ceramic crowns failed prior to the follow-up examination

${ }^{4}$ Significant difference between baseline and 2-year and 5-year investigation $(p \leq 0.05)$

\section{Discussion}

After a mean observational period of 5 years, in the present study, a significant effect of the MMT of chairsidefabricated ZLS-ceramic partial crowns on the survival rate could be detected. A survival rate of $100 \%$ at an MMT of $0.75-1.0 \mathrm{~mm}$ was determined, while the 5-year survival rate of partial crowns with an MMT of $<0.75 \mathrm{~mm}$ was significantly reduced to $83 \%$ (95\% CI: $0.71-0.96)$. This effect has not been documented in clinical trials yet. Apart from the MMT, the position of the restorations (premolar vs. molar) had a significant effect on the 5-year success rate of chairside fabricated ZLS partial crowns. Restorations luted with SAC showed a pronounced tendency for an increased risk for a loss of retention (HR: 9.2). These findings are in good accordance with the results of other clinical studies on ceramic partial crowns [5-8, 29-31].

For the interpretation of the results of the present study, it should be considered that the findings of the clinical investigation are influenced by a variety of variables, e.g., study design (prospective vs. retrospective, fabrication technique, evaluation criteria, observational period). Therefore, the results of the present study should preferably be compared with those of clinical studies on chairside-fabricated all-ceramic partial crowns with a similar design (prospective), using comparable survival and success criteria, and reporting a comparable observational period $[18,19,29-31$, 34-36].

Federlin et al. (2010) documented a survival rate of $88.8 \%$ after 5.5 years for chairside-fabricated feldspathic ceramic partial crowns (Vita MK II, Vita Zahnfabrik, Bad Säckingen, Germany) with an MMT of $1.5 \mathrm{~mm}$. Material fracture was the most frequent cause of failure [30]. This is in good accordance with the findings of another prospective clinical trial evaluating the clinical performance of chairsidefabricated feldspathic ceramic partial crowns luted with an
SAC. At the 3-year recall, 7 out of 12 complete failures were attributed to catastrophic material fractures [31].

For chairside-fabricated ceramic partial crowns fabricated from a leucite-reinforced ceramic material (ProCAD, Ivoclar Vivadent, Schaan, Liechtenstein), a 3-year survival rate of 97\% was reported [34]. From another prospective clinical trial on chairside-fabricated leucite-reinforced glass-ceramic onlays (Empress CAD, Ivoclar Vivadent, Schaan, Liechtenstein), a material fracture rate of $6.7 \%$ during a 5-year observational period was reported [36]. In both studies, the restorations were fabricated with a minimum material thickness of 1.2-1.5 $\mathrm{mm}$ according to the manufacturers' recommendations. Interestingly, an additional study on conventionally fabricated partial crowns made from the same glass-ceramic material reported that a mere reduction in the MMT to 1.0-1.4 $\mathrm{mm}$ caused a significant increase in fracture-related loss after a mean observational period of 3 years [37].

To date, a limited number of clinical trials with maximum observational periods of 3 years have evaluated chairsidefabricated ZLS-ceramic partial crowns [17-19]. From a practice-based study evaluating chairside-fabricated ZLSceramic partial crowns (Celtra Duo, Dentsply Sirona, Bensheim Germany), a 3-year survival rate of $99 \%$ (CI-95\%: 0.97-1) was reported. The restorations were adhesively luted with TEC materials and fabricated with an MMT of $1.5 \mathrm{~mm}$. Furthermore, 2-year results from the present study have already been published. During this follow-up, 2 material fractures required the replacement of 2 restorations from group 1 (2-year survival rate group 1: 94\% CI-95\%: $0.85-1)$ ). No losses occurred in group 2 (2-year survival rate group 2: 100\%) [18].

For the 5-year follow-up reported in the present study, no fracture-related losses were documented with a moderate reduction relative to the manufacturer-recommended MMT (0.75-1.0 mm), independent of the applied 
cementation technique. Thus, the survival rate was in the same range as that of chairside-fabricated partial crowns made of other ZLS-ceramic materials and conventionally fabricated partial crowns made of lithium disilicate ceramic materials with an MMT of $1.5 \mathrm{~mm}[19,34]$. Fracture-related losses occurred only after a massive reduction relative to the recommended MMT $(0.5-0.74 \mathrm{~mm})$.

In this group, the survival rate after a mean observational period of 5 years was $83 \%$ due to 5 fractures. Statistical analysis showed that the survival rate was dependent on the MMT of the partial crowns (MMT of $0.5-0.74 \mathrm{~mm}$ vs. $0.75-1.0 \mathrm{~mm}, p=0.0292$ ). The evaluated HR of 0.09 showed an 11.1-fold reduced risk of material fracture, demonstrating a significant influence of the MMT on the fracture-related failure of chairside-fabricated partial crowns made of a ZLS-ceramic material.

It was taken into account that due to necessary occlusal adjustments the finally cemented restoration could be thinner than the dimensions measured from the construction data. Nevertheless, in the course of the present study, only minor adjustments of the cemented restoration were carried out with fine grit size diamond instruments and were mainly related to the cusp areas. Therefore, this seems to be a low risk for a bias of the results.

The effect of the MMT on the fracture strength of ceramic crowns and partial crowns has been evaluated only in several in vitro studies [20-23] and a limited number of clinical studies [18, 27]. Based on these investigations, it was postulated that for high-strength glass-ceramic materials (e.g., lithium-disilicate ceramics or ZLS-ceramics), the MMT can be reduced to $1.0 \mathrm{~mm}$.

Considering the limitations, the results of the present study can be interpreted as an initial clinical verification that for high-strength glass-ceramic materials, the occlusal MMT can be reduced to $1.0 \mathrm{~mm}$ without an increase in the rate of fracture-related failures. This represents a new finding not reported from a clinical trial yet. However, to specify a defined limit for an MMT leading to fracturerelated failures, additional studies with larger populations and longer observational periods are required [29, 32].

Although all complete failures in the present study occurred exclusively on molars, the statistical analysis failed to determine a statistically significant effect of the position of the restoration on the survival rate. This might be related to a small subgroup with a skew distribution of the restorations (23 premolar restorations/36 molar restorations) and a limited number of observations. These aspects are indicators that the study was underpowered. Therefore, a post hoc power analysis was performed for the risk factor "position of the restoration." Post hoc sample size calculation based on the incidence of prosthesis failures revealed that 50 restorations would be needed per group to confirm a statistically significant difference between the molar and premolar restorations 5 years after prosthesis insertion.

Clinical interventions for maintaining the function of the restorations during the 5-year observational period were mainly required due to a loss of retention. Endodontic treatment was needed for one out of 54 restorations (complication rate $1.9 \%$ ) during a 5 -year functional period. No secondary caries were observed. These findings are in good accordance with other clinical studies reporting on the clinical performance of adhesively luted ceramic partial crowns [18, 29-31].

A loss of retention $(n=4)$ occurred only in restorations that were luted with SAC, while restorations inserted with TEC showed no loss of retention. Statistical analysis revealed a pronounced tendency toward an increased risk $(\mathrm{HR}=9.2)$ of retention loss in restorations inserted with SAC. However, this effect was not statistically significant $(p=0.0527)$. Post hoc sample size calculation based on the incidence of loss of retention revealed that the sample size of the subgroups was too small to confirm a statistically significant difference between the two cementation techniques.

Nevertheless this observation is in good accordance with the results of other clinical studies showing increased rates of retention loss in chairside-fabricated partial crowns inserted with SAC [29, 31, 35].

A possible explanation of the increased debonding rate can be seen in the fact that the bond strength of self-adhesive resin cements to the tooth surface (enamel and dentin) was reported to be inferior to conventional composite resin cements [38-40]. This fact is of importance as in the present clinical study, a non-retentive preparation design for the partial crowns was used. For these preparations where dentin was predominant, cements with higher bond strength as total-etch or self-etch resin cements may provide a better protection against a loss of retention [29, 35].

In the present study, all restorations could be recemented after the loss of retention. In contrast, in clinical studies on chair-side fabricated restorations made from feldspathic materials, the loss of retention was mostly related to restoration fracture, so no recementation was possible [29, 31, 35]. This observation can be explained by the increased mean flexural strength of the applied ZLS-ceramic materials in comparison with feldspathic ceramic materials [10-13].

Regarding the success rate in the present study a significant influence of the tooth position (premolar vs. molar) could be detected. Restorations placed on a molar revealed a 5.26-fold increased risk for a failure or a clinical intervention to maintain function. This is in good accordance with the findings of other clinical trials and can be attributed to higher occlusal load in the molar area compared to the premolar area [5-8].

With respect to clinical changes (USPHS criteria) over time, statistically significant differences were determined 
between baseline and the 5-year recall regarding the criteria for marginal adaptation and marginal discoloration. Both criteria showed a statistically significant increase in bravo ratings over time for both cementation techniques, along with a statistically significant decrease in alpha ratings $(p \leq 0.05)$. This is in accordance with the findings of previous studies reporting USPHS criteria changes in the same range for CAD/CAM-fabricated partial crowns from different materials [18, 29-31].

An explanation for this observation can be seen in the wear of the luting material and an increased width of the luting space of the CAD/CAM-fabricated partial crowns [29-31]. Furthermore, for the SAC used in the present study, wear of the resin matrix and loss of fillers resulting in an increase in roughness were reported as reasons for increased staining capacity over time [31]. Additionally, individual patient parameters (diet, smoking habits, and oral home care procedures) must be taken into account [30, 31].

The results of the post hoc power analysis indicated that the study was-due to the small study population and limited number of events-underpowered. Therefore, possible effects of some of the evaluated variables on the survival and success rates could not be verified by the statistical analysis.

This represents a major limitation of the present study. In particular, the importance of a prolonged observational period to detect possible risk factors influencing the clinical performance can be seen when the results of the present study are compared with the previous results reported from the same study population [18]. After a mean observational period of 2 years, two complete failures were reported. The number of failures increased during the following 3 years to 5 failures. Due to the limited number of events, a risk analysis based on the 2-year results was not possible. With the prolonged observational period, the number of events increased and allowed for a sound statistical analysis. Thus, the abovementioned limitations of the studies could be partially compensated by the prolonged observational period. The importance of prolonged observational periods ( $\geq 5$ years) has been described in other prospective studies as well [29, 31].

Another limitation is the deviation from the original study protocol caused by an operating error during the construction process that led to a violation of the MMT $(1.0 \mathrm{~mm})$ recommended by the manufacturer. Thus, the restorations were not randomized regarding this parameter. This is a limitation for a clinical research project. On the other hand, this inadvertent deviation from the study protocol provided important evidence on the possible clinical impact of errors in the fabrication process of chairside-fabricated restorations. To the best of the authors' knowledge, this impact has not yet been documented in the literature.

Despite these limitations, the present study provides important information on the clinical safety and performance of chairside-fabricated partial crowns because it offers clinical data for relatively new ZLS-ceramic materials, which have been evaluated in a limited number of clinical studies with shorter observational periods ( $\leq 3$ years) only [17-19]. Moreover, this study provides the first clinical data on chairside-fabricated partial crowns with a reduced MMT, generating important information to verify the manufacturers' recommendations based on in vitro data only for a less invasive preparation design for high-strength glass-ceramic restoration (MMT: $1.0 \mathrm{~mm}$ ). Furthermore, the study verifies the relevance of the cementation technique and restoration position for the long-term evaluation of all-ceramic partial crowns, as already documented in other clinical studies [5-8, 29, 31, 35].

However, due to the limited sample size, a general clinical recommendation must be made with caution, as the present study was underpowered to detect significant effects of potential risk factors other than the MMT and tooth position. Therefore, further clinical studies with longer observational periods and larger sample sizes are needed.

\section{Conclusions}

Chairside-fabricated ZLS-ceramic partial crowns showed a 5 -year survival rate of $100 \%$ with a moderate reduction relative to the manufacturer's recommendations regarding the occlusal MMT (0.75-1.0 mm), independent of the cementation technique. A further reduction in the occlusal MMT $(0.5-0.74 \mathrm{~mm})$ led to a significant increase in losses caused by material fractures (HR: 11.1). The success probability of ZLS-ceramic partial crowns was significantly influenced by the tooth position, with molar restorations demonstrating a 5.26-fold increased risk for a failure or need for a clinical intervention.

Cementation of the ZLS-ceramic partial crowns with SAC showed a pronounced tendency (HR: 9.2) toward an increased rate of retention loss compared to restorations luted with a TEC material. Clinical evaluations with longer observational periods and larger sample sizes are essential for further examination of these potential influencing factors.

Funding Open Access funding enabled and organized by Projekt DEAL. This research received a specific grant from Vita Zahnfabrik, Bad Säckingen, Germany. The funding source had no influence in the collection, analysis, and interpretation of data, in writing the report, and the decision to submit the article for publication.

\section{Declarations}

Ethics approval All procedures performed in studies involving human participants were in accordance with the ethical standards of the insti- 
tutional and/or national research committee and with the 1964 Helsinki Declaration and its later amendments or comparable ethical standards. The study design was approved by the local ethics committee.

Consent to participate Informed consent was obtained from all individual participants included in the study.

Conflict of interest The authors declare no competing interests.

Open Access This article is licensed under a Creative Commons Attribution 4.0 International License, which permits use, sharing, adaptation, distribution and reproduction in any medium or format, as long as you give appropriate credit to the original author(s) and the source, provide a link to the Creative Commons licence, and indicate if changes were made. The images or other third party material in this article are included in the article's Creative Commons licence, unless indicated otherwise in a credit line to the material. If material is not included in the article's Creative Commons licence and your intended use is not permitted by statutory regulation or exceeds the permitted use, you will need to obtain permission directly from the copyright holder. To view a copy of this licence, visit http://creativecommons.org/licenses/by/4.0/.

\section{References}

1. Veneziani M (2017) Posterior indirect adhesive restorations: updated indications and the morphology driven preparation technique. Int J Esthet Dent 12:204-230

2. Edelhoff D, Ahlers MO (2018) Occlusal onlays as a modern treatment concept for the reconstruction of severely worn occlusal surfaces. Quintessence Int 49:521-533. https://doi.org/10.3290/j. qi.a40482

3. Spitznagel FA, Boldt J, Gierthmuehlen PC (2018) CAD/CAM ceramic restorative materials for natural teeth. J Dent Res 97:1082-1091. https://doi.org/10.1177/0022034518779759

4. Politano G, van Meerbeek B, Peumans M (2018) Nonretentive bonded ceramic partial crowns: concept and simplified protocol for long-lasting dental restorations. J Adhes Dent 20:495-510. https://doi.org/10.3290/j.jad.a41630

5. Abduo J, Sambrook RJ (2018) Longevity of ceramic onlays: a systematic review. J Esthet Restor Dent 30:193-215. https://doi. org/10.1111/jerd.12384

6. Mangani F, Marini S, Barabanti N, Preti A, Cerutti A (2015) The success of indirect restorations in posterior teeth: a systematic review of the literature. Minerva Stomatol 64:231-240

7. Morimoto S, Rebello de Sampaio FBW, Braga MM, Sesma N, Özcan M (2016) Survival Rate of resin and ceramic inlays, onlays, and overlays: a systematic review and meta-analysis. J Dent Res 95:985-994. https://doi.org/10.1177/0022034516652848

8. Otto T (2017) Up to 27-years clinical long-term results of chairside Cerec 1 CAD/CAM inlays and onlays. Int J Comput Dent 20:315-329

9. Bakeman EM, Rego N, Chaiyabutr Y, Kois JC (2015) Influence of ceramic thickness and ceramic materials on fracture resistance of posterior partial coverage restorations. Oper Dent 40:211-217. https://doi.org/10.2341/12-459-L

10. Elsaka SE, Elnaghy AM (2016) Mechanical properties of zirconia reinforced lithium silicate glass-ceramic. Dent Mater 32:908-914. https://doi.org/10.1016/j.dental.2016.03.013

11. Furtado de Mendonca A, Shahmoradi M, de Gouvêa CVD, de Souza GM, Ellakwa A (2019) Microstructural and mechanical characterization of CAD/CAM materials for monolithic dental restorations. J Prosthodont 28:e587-e594. https://doi.org/10.1111/ jopr.12964

12. Hamza TA, Sherif RM (2019) Fracture resistance of monolithic glass-ceramics versus bilayered zirconia-based restorations. J Prosthodont 28:e259-e264. https://doi.org/10.1111/jopr.12684

13. Sen N, Us YO (2018) Mechanical and optical properties of monolithic CAD-CAM restorative materials. J Prosthet Dent 119:593599. https://doi.org/10.1016/j.prosdent.2017.06.012

14. Sulaiman TA, Abdulmajeed AA, Delgado A, Donovan TE (2019) Fracture rate of 188695 lithium disilicate and zirconia ceramic restorations after up to 7.5 years of clinical service: a dental laboratory survey. J Prosthet Dent 123:807-801. https://doi.org/10. 1016/j.prosdent.2019.06.011

15. Belli R, Petschelt A, Hofner B, Hajtó J, Scherrer SS, Lohbauer U (2016) Fracture rates and lifetime estimations of CAD/CAM all-ceramic restorations. J Dent Res 95:67-73. https://doi.org/10. 1177/0022034515608187

16. Edelhoff D, Güth JF, Erdelt K, Brix O, Liebermann A (2019) Clinical performance of occlusal onlays made of lithium disilicate ceramic in patients with severe tooth wear up to 11 years. Dent Mater 35:1319-1330. https://doi.org/10.1016/j.dental.2019.06. 001

17. Zimmermann M, Koller C, Mehl A, Hickel R (2017) Indirect zirconia-reinforced lithium silicate ceramic CAD/CAM restorations: preliminary clinical results after 12 months. Quintessence Int 48:19-25. https://doi.org/10.3290/j.qi.a37017

18. Rinke S, Brandt A, Hausdoerfer T, Leha A, Ziebolz D (2020) Clinical evaluation of chairside-fabricated partial crowns made of zirconia-reinforced lithium silicate ceramic-2-year-results. Eur J Prosthodont Restor Dent 28:36-42. https://doi.org/10.1922/ EJPRD_2001Rinke07

19. Rinke S, Pfitzenreuter T, Leha A, Roediger M, Ziebolz D (2020) Clinical evaluation of chairside-fabricated partial crowns composed of zirconia-reinforced lithium silicate ceramics: 3-year results of a prospective practice-based study. J Esthet Restor Dent 32:226-235. https://doi.org/10.1111/jerd.12542

20. Al-Akhali M, Chaar MS, Elsayed A, Samran A, Kern M (2017) Fracture resistance of ceramic and polymer-based occlusal veneer restorations. J Mech Behav Biomed Mater 74:245-250. https:// doi.org/10.1016/j.jmbbm.2017.06.013

21. Choi S, Yoon H-I, Park E-J (2017) Load-bearing capacity of various CAD/CAM monolithic molar crowns under recommended occlusal thickness and reduced occlusal thickness conditions. J Adv Prosthodont 9:423-431. https://doi.org/10.4047/jap.2017.9. 6.423

22. Bergamo ETP, Bordin D, Ramalho IS, Lopes ACO, Gomes RS, Kaizer M et al (2019) Zirconia-reinforced lithium silicate crowns: effect of thickness on survival and failure mode. Dent Mater 35:1007-1016. https://doi.org/10.1016/j.dental.2019.04.007

23. Zimmermann M, Egli G, Zaruba M, Mehl A (2017) Influence of material thickness on fractural strength of CAD/CAM fabricated ceramic crowns. Dent Mater J 36:778-783. https://doi.org/10. 4012/dmj.2016-296

24. Sampaio FBWR, Özcan M, Gimenez TC, Moreira MSNA, Tedesco TK, Morimoto S (2019) Effects of manufacturing methods on the survival rate of ceramic and indirect composite restorations: a systematic review and meta-analysis. J Esthet Restor Dent 31:561-571. https://doi.org/10.1111/jerd.12555

25. de Souza Melo G, Batistella EÂ, Bertazzo-Silveira E, Simek Vega Gonçalves TM, Mendes de Souza BD, Porporatti AL et al (2018) Association of sleep bruxism with ceramic restoration failure: a systematic review and meta-analysis. J Prosthet Dent 119:354362. https://doi.org/10.1016/j.prosdent.2017.07.005

26. van de Sande FH, Collares K, Correa MB, Cenci MS, Demarco FF, Opdam N (2016) Restoration survival: revisiting patients' risk 
factors through a systematic literature review. Oper Dent 41:S7S26. https://doi.org/10.2341/15-120-LIT

27. Hamoudi W, Trulsson M, Svensson P, Smedberg JI (2020) Longterm results of a randomized clinical trial of 2 types of ceramic crowns in participants with extensive tooth wear. J Prosthet Dent S0022-3913(20):30651-X

28. Ahlers MO, Mörig G, Blunck U, Hajtó J, Pröbster L, Frankenberger R (2009) Guidelines for the preparation of CAD/CAM ceramic inlays and partial crowns. Int J Comput Dent 12:309-325

29. Baader K, Hiller K-A, Buchalla W, Schmalz G, Federlin M (2016) Self-adhesive luting of partial ceramic crowns: selective enamel etching leads to higher survival after 6.5 years in vivo. J Adhes Dent 18:69-79. https://doi.org/10.3290/j.jad.a35549

30. Federlin M, Hiller K-A, Schmalz G (2010) Controlled, prospective clinical split-mouth study of cast gold vs. ceramic partial crowns: 5.5 year results. Am J Dent 23:161-167

31. Federlin M, Hiller K-A, Schmalz G (2014) Effect of selective enamel etching on clinical performance of CAD/CAM partial ceramic crowns luted with a self-adhesive resin cement. Clin Oral Investig 18:1975-1984. https://doi.org/10.1007/ s00784-013-1173-2

32. Anusavice KJ (2012) Standardizing failure, success, and survival decisions in clinical studies of ceramic and metal-ceramic fixed dental prostheses. Dent Mater 28:102-111. https://doi.org/10. 1016/j.dental.2011.09.012

33. Gerds TA, Qvist V, Strub JR, Pipper CB, Scheike TH, Keiding N (2009) Failure time analysis. In: Lesaffre E, Feine J, LeRoux B (eds) Statistical and methodological aspects of oral health research. John Wiley and Sons, West Sussex, pp 259-278

34. Guess PC, Strub JR, Steinhart N, Wolkewitz M, Stappert CFJ (2009) All-ceramic partial coverage restorations-midterm results of a 5-year prospective clinical splitmouth study. J Dent 37:627637. https://doi.org/10.1016/j.jdent.2009.04.006
35. Vogl V, Hiller K-A, Buchalla W, Federlin M, Schmalz G (2016) Controlled, prospective, randomized, clinical split-mouth evaluation of partial ceramic crowns luted with a new, universal adhesive system/resin cement: results after 18 months. Clin Oral Investig 20:2481-2492. https://doi.org/10.1007/s00784-016-1779-2

36. Fasbinder DJ, Neiva GF, Heys D, Heys R (2020) Clinical evaluation of chairside computer assisted design/computer assisted machining nano-ceramic restorations: five-year status. J Esthet Restor Dent 32:193-203. https://doi.org/10.1111/jerd.12516

37. Murgueitio R, Bernal G (2012) Three-year clinical follow-up of posterior teeth restored with leucite-reinforced ips empress onlays and partial veneer crowns. J Prosthodont 21:340-345. https://doi. org/10.1111/j.1532-849X.2011.00837.x

38. Lührs AK, Guhr S, Günay H, Geurtsen W (2010) Shear bond strength of self-adhesive resins compared to resin cements with etch and rinse adhesives to enamel and dentin in vitro. Clin Oral Investig 14(2):193-199. https://doi.org/10.1007/ s00784-009-0279-z

39. Poitevin A, De Munck J, Van Ende A, Suyama Y, Mine A, Peumans M, Van Meerbeek B (2013) Bonding effectiveness of selfadhesive composites to dentin and enamel. Dent Mater 29(2):221230. https://doi.org/10.1016/j.dental.2012.10.001

40. Peumans M, Voet M, De Munck J, Van Landuyt K, Van Ende A, Van Meerbeek B (2013) Four-year clinical evaluation of a self-adhesive luting agent for ceramic inlays. Clin Oral Investig 17(3):739-750. https://doi.org/10.1007/s00784-012-0762-9

Publisher's note Springer Nature remains neutral with regard to jurisdictional claims in published maps and institutional affiliations. 